\title{
Die berufliche Gleichstellung von Frauen braucht rechtliche Regulierung
}

Zweifellos: Die Gleichstellung der Frauen hat Fortschritte gemacht. Frauen haben sich mittlerweile viele Terrains erobert, die ihnen früher verschlossen waren. Und sie stehen heute auf anderen rechtlichen Füßen als noch vor hundert Jahren. Doch Fakt ist auch: Die Emanzipation kriecht im Schneckentempo. Und sie trifft stets erneut auf Widerstände, die sie vom Ziel abbringen, ja oftmals auch zurückwerfen. Das schürt auf Dauer nicht nur die Ungeduld, sondern lässt die Gedanken immer wieder um die Fragen kreisen, warum dies so ist und an welchen Stellschrauben wie gedreht werden könnte, um in Sachen Gleichberechtigung bessere, schnellere und dauerhaftere Erfolge als bisher zu erzielen. ${ }^{1}$

\section{DIE AKTUELLE (RECHTS-)LAGE}

Unsere Verfassung ist eindeutig: Ein Diskriminierungsverbot ist in Art. 3 Abs. 3 GG ebenso verankert wie das in das Grundgesetz implementierte Gebot, dass Männer und Frauen gleichberechtigt sind (Art. 3 Abs. 2 GG).

Damit ist der Gesetzgeber zunächst gehalten, im Recht vorhandene Ungleichbehandlungen zu beseitigen. Doch schon hier hat sich gezeigt, dass dieser sich gerade im Hinblick auf die Gleichheit der Geschlechter nicht sogleich bemüßigt fühlte, Frauen diskriminierendes Recht aufzuheben. Es bedurfte der vielfachen Anmahnung durch das Bundesverfassungsgericht, bis das Gesetzesrecht Stück für Stück von unmittelbar diskriminierenden Regeln befreit wurde.

Doch der Widerstand allein schon gegen das Benachteiligungsverbot ist damit keineswegs aufgegeben worden. Vor allem im Arbeitsleben hat er Beharrlichkeit erwiesen. Mittlerweile gut getarnt und eingepackt in die der political correctness geschuldete Versicherung, dass es eine pure Selbstverständlichkeit sei, Männer und Frauen gleich zu behandeln, bricht er sich hier immer dann urwüchsig Bahn, wenn die Gefahr gewittert wird, dass durch Rechtsetzung die geübte Praxis, dem Gleichbehandlungsgebot im Tatsächlichen dennoch ein Schnippchen zu schlagen, eingeengt werden könnte. So geschehen, als 1980 auf Druck Europas ein ausdrückliches Gleichbehandlungsgebot ins Arbeitsrecht Eingang fand und sich dagegen ein Heulen und Zähneklappern erhob, das den Untergang der Privatautonomie beklagte. Und ein Vierteljahrhundert später, als dieses Gebot lediglich in das wiederum von Europa angestoßene Allgemeine Gleichbehandlungsgesetz transferiert werden sollte, formierte sich dagegen erneut massiver Protest. Von unzumutbaren Gängeleien und Belastungen war die Rede, denen die Wirtschaft dadurch ausgesetzt sei. Das ist schon mehr als erstaunlich, hat doch die langjährige Erfahrung mit diesem Gleichbehandlungsgebot gezeigt, dass es der männlichen Personalauslese faktisch nicht im Wege steht, ihr nur eine geschickte Begründung abverlangt. Und nach wie vor belegt die Statistik, dass es keine größeren Umstände bereitet, Frauen und Männer ungleich zu entlohnen.

Aber selbst die formal rechtliche Gleichbehandlung hebt die sozial tief verankerten ungleichen Lebenssituationen von Männern und Frauen nicht auf. Im Gegenteil, sie kann, wenn sie sich am männlichen Lebenszuschnitt ausrichtet, reale Ungleichheiten sogar zementieren. Man nennt dies mittelbare Diskriminierung. Eigentlich liegt diese Erkenntnis schon dem verfassungsrechtlichen Postulat der Gleichberechtigung zugrunde. Denn weshalb hätte es des Kampfes um seine Aufnahme ins Grundgesetz bedurft, wenn mit Art. 3 Abs. 2 GG nichts anderes zur Verpflichtung gemacht werden sollte als das, was schon dessen Abs. 3 mit seinem Verbot der Diskriminierung wegen des Geschlechts vorschreibt? Doch nur, weil damit auch die Beseitigung der Ungleichheit in der Wirklichkeit und die Herstellung von realer Gleichheit angemahnt werden sollte. Aber dieser, auf Veränderung abzielende Impetus des Gleichberechtigungsgebots wurde zunächst stillgeschwiegen und dann, als Frauen in den 1970er Jahren auf die Einlösung dieses Gebots pochten, auf das Heftigste bestritten. Man brandmarkte die Forderung als umstürzlerischen Angriff auf gewichtige Grundrechtspositionen, Werthaltungen und Strukturentscheidungen unserer Verfassung. Die Herbeiführung von Gleichheit durch spezifische Förderung von Frauen, das Ausgleichen von Benachteiligung durch Bevorzugung - sie wurden als verfassungswidriger Eingriff in die Unternehmerfreiheit, ja in die gesamte Wirtschaftsverfassung, als Verstoß gegen das Prinzip der Bestenauslese und nicht zuletzt als unzulässige Diskriminierung von Männern betrachtet.

Und obwohl das Verfassungsgericht im Streit darüber schon in ein paar Entscheidungen signalisiert hatte, dass Frauen begünstigende Regelungen durchaus mit der Verfassung in Einklang stehen können, erklang nochmals großes Geschrei, als Frauen Anfang der 1990er Jahre im Rahmen der Verfassungsreformdebatten, die anlässlich der Wiedervereinigung Deutschlands geführt wurden, reklamierten, in Art. 3 Abs. 2 GG ausdrücklich klarzustellen, dass Frauenförderung nicht nur erlaubt, sondern geboten

Der Beitrag ist die stark gekürzte Fassung eines
Vortrags zum Thema "Berufliche Gleichstellung
von Frauen - Notwendigkeit und Formen einer
Regulierung“, den die Autorin auf dem WSI-
Herbstforum "Chancen und Grenzen von Regu-
lierung " am 3./4.12.2009 in Berlin gehalten hat.

Christine Hohmann-Dennhardt, Dr., Richterin am Bundesverfassungsgerichts. Arbeitsschwerpunkte: Arbeits-, Sozial-, Familien-, Verfassungsrecht. e-mail: uempl@Bundesverfassungsgericht.de 
ist. Erzielt wurde ein Teilerfolg. Die Kompromissformel, die gefunden wurde und seither das Gleichberechtigungsgebot ergänzt, lautet: „Der Staat fördert die tatsächliche Durchsetzung der Gleichberechtigung von Frauen und Männern und wirkt auf die Beseitigung bestehender Nachteile hin". Wie sehr an diesem Satz gefeilt und welche raffinierte juristische Rabulistik bemüht wurde, damit Befürworterinnen wie Widersacher ihren Standpunkt darin wiederfinden konnten, zeigt der damalige Abschlussbericht der Gemeinsamen Verfassungskommission.

\section{GRABENKÄMPFE IN DER ARBEITSWELT}

Doch vor allem in der Arbeitswelt ist die Verteidigungsfront männlicher Macht nach wie vor gut aufgestellt, insbesondere dort, wo in den Führungsetagen die Marschrouten ausgegeben werden und die Personalauslese erfolgt. Hier will man sich nicht auch noch das Heft aus der Hand nehmen lassen. Zwar kann und will man den Frauen nicht mehr den Zutritt ins Berufsleben versagen. Denn sie werden gebraucht und für bestimmte Dienste und Tätigkeiten als zumeist billigere Arbeitskräfte gerne zum Einsatz gebracht. Doch als Konkurrentinnen um die Chefsessel sind sie unerwünscht. Sollten hier dennoch einzelne weibliche Einsprengsel zu finden sein, ist dies weniger Resultat der Offenheit gegenüber weiblicher Begabung, sondern dient eher der Kaschierung des männlichen Machterhalts. Die Reihen fest geschlossen, so lautet die Devise. Deshalb die Pflege der männlichen Seilschaften. Deshalb das beharrliche Festhalten an Arbeitsverhältnissen, die auf Männer - in Subtraktion familiärer Pflichten und Addition hausfraulicher Unterstützung - zugeschnitten sind. Dazu gehören das stetige Hochfahren des Leistungsdrucks, die Ausdehnung der Arbeitszeiten und das Einfordern allzeitiger Flexibilität wie Einsatzbereitschaft. Da können Frauen schwerer mithalten und hochkommen, wenn ihnen die Aufgabe überlassen bleibt, Beruf und Familie unter einen Hut zu bringen. Männer dagegen passen sich gefügig diesem immer höher geschraubten Anforderungsprofil an und strecken sich nach der Decke, selbst wenn ihnen der Sinn nach mehr familiärem Leben steht, ist doch die Arbeitswelt für sie verinnerlichte Vorsehung und maßgeblicher Ort, an dem sie Wertschätzung, Erfolg und damit Selbstbestätigung ihrer Männlichkeit zu finden glauben.
Aber es geht bei diesem Abwehrgefecht nicht allein um die Verteidigung der Führungsebenen gegen weibliche Infiltration. Man fürchtet viel Schlimmeres: dass die gesamte Statik der Arbeitswelt, die auch die Profite trägt, in sich zusammenfallen könnte, wenn Frauen vermehrt das Sagen hätten und dann vielleicht damit beginnen würden, die Arbeitsstrukturen zu verändern, um sie mit dem Wunsch nach Vereinbarkeit von Beruf und Familie, von Arbeit und Privatleben, kompatibel zu machen. Denn dann müssten die Arbeitszeiten und -rhythmen neu ausgerichtet, die Zusammenarbeit neu strukturiert, die Leistungsbewertungen anders gewichtet, die Entlohnung angeglichen und die Infrastrukturen an neue Bedarfe angepasst werden. Die stärkere Berücksichtigung sozialer Bedürfnisse aber widerspricht den Kapitalinteressen nach steter Steigerung der Arbeitsproduktivität im gewohnten Stil und damit der Gewinnmaximierung. Die Verteidigung der Männerbastionen in den Führungsetagen dient also neben der männlichen Selbstgefälligkeit vor allem dazu, die Macht des Kapitals zu behaupten und seine Interessen am Erhalt der bestehenden Arbeitsstrukturen zu wahren. Darum so vehementer Widerstand.

\section{WAS TUN?}

Welche Schlüsse sind aus dieser Zustandsund Ursachenbeschreibung nun aber zu ziehen? Zunächst einer ganz gewiss: Es führt nicht weiter, bei der Herstellung von Gleichberechtigung auf die Freiwilligkeit derjenigen zu setzen, die im Eigeninteresse die Arbeitswelt, so wie sie funktioniert, gerade erhalten wollen. Es müssen also gesetzliche Regeln her, die dazu anhalten, das unternehmerische Handeln und Entscheiden am Ziel der Gleichberechtigung auszurichten.

Dabei lehrt uns die Erfahrung, dass abstrakte Verbotsnormen zwar Signalfunktion haben und sensibilisieren können. Doch sie laufen zumeist leer, weil der Nachweis ihrer Verletzung im Einzelfall nur schwer gelingt. Es lassen sich stets Gründe finden, warum der Mann - nicht etwa qua Geschlecht, sondern qua Qualifiktion - der bessere Bewerber sei.

\section{BEGRENZTE WIRKUNGEN VON SOFT-LAW-REGELUNGEN}

Wenden wir uns beim Durchforsten des rechtlichen Instrumentenkastens deshalb den Normen zu, die konkrete Handlungs- und Zielvorgaben beinhalten. Mit ihnen kann z. B. vorgeschrieben werden, in Förderplänen festzulegen, in welcher Zeit und in welchem Ausmaß der Frauenanteil in den jeweiligen Arbeitsbereichen und -hierarchien zu steigern ist. Rechtlich ist dies durchaus darstellbar. Doch inwieweit solche Vorgaben tatsächlich die gewünschten Änderungen bewirken, hängt nicht nur vom notwendigen, aber oftmals nicht vorhandenen Impetus der Personalentscheider $\mathrm{ab}$, sondern auch von Faktoren, die rechtlich nicht steuerbar sind. So wissen wir aufgrund der Erfahrungen im öffentlichen Dienst, dass Personalabbau durch Wegfall von Stellen ein solches Konzept zum Erlahmen bringt. Wo es keine oder nur sehr wenige Stellen zu besetzen gibt, können auch Frauen darauf nicht Platz nehmen. Und geht es bei der Vergabe der wenigen Stellen nach dem Prinzip der „Bestenauslese", erhalten subjektive Kriterien der Personalentscheider Gewicht. Das bleibt unbefriedigend, macht aber dennoch das Unterfangen solch rechtlich angeordneter Förderpläne keineswegs obsolet. Denn so wird zumindest offengelegt, wie groß die Benachteiligungslage und entsprechend der Förderbedarf von Frauen in den einzelnen Beschäftigungsbereichen und - ebenen real noch ist. Und es zwingt zur Erklärung, weshalb das gesteckte Ziel verfehlt worden ist und was unternommen wird, um es beim erneuten Anlauf zu erreichen. Hier voranzukommen, gleicht allerdings oftmals der Arbeit des Sysiphos, wovon Frauenbeauftragte ein Lied singen können.

Noch mühseliger ist es dort, wo gestecktes Ziel nicht die quantitative Erhöhung des Frauenanteils an den Beschäftigten ist, sondern es um die qualitative Verbesserung der Situation und Chancen von Frauen geht. Zwar könnte man auch hier z. B. vorgeben, wie groß der Anteil von Frauen in proportionaler Umkehrung ihres Anteils an der Beschäftigtenzahl bei Fortbildungsveranstaltungen sein muss. Oder man könnte errechnen, wie groß der Bedarf an betrieblichen Kinderbetreuungsplätzen ist. Wenn aber innerbetriebliche Fortbildungsveranstaltungen nicht stattfinden oder ein Angebot der Kinderbetreuung aus finanziellen Gründen unterbleibt, dann lassen sich solche Fördermaßnahmen durch rechtliche Zielvorgaben nicht erzwingen. Und gesetzlich vorzugeben, dass z.B. ein bestimmter Prozentsatz der zur Verfügung stehenden Mittel für frauenfördernde Maßnahmen auszugeben ist, würde 
sofort als Eingriff in die Unternehmensfreiheit gebrandmarkt. Allerdings könnte eine besondere steuerliche Absetzbarkeit solcher Ausgabepositionen einen sanften Druck ausüben und dazu bewegen, „freiwillig" doch etwas mehr Mittel auf solche Maßnahmen zu verwenden. Doch dies sind nur vage Hoffnungen.

Es ist kein falscher, aber vielleicht $\mathrm{zu}$ verengter Ansatz, zur Beförderung der Gleichstellung vornehmlich nach rechtlichen Mitteln zu suchen, die quasi von unten her auf die Prozeduren der Personalauslese und -qualifizierung steuernd einwirken sollen. Das funktioniert, wie die Erfahrung lehrt, nur langsam und endet bei Stellenbesetzungen in den mittleren Hierarchien. Mag sein, dass es Frauen bisweilen auch an Mut oder Motivation mangelt, in Höhen aufzusteigen, wo mit harten Bandagen gekämpft wird. Aber angesichts der mittlerweile bestehenden Vielzahl von bestens qualifizierten Frauen erklärt dies allein nicht die immer noch marginale und stagnierende Zahl von Frauen in Führungspositionen. Nein, ausschlaggebend hierfür ist, wie aufgezeigt, der mangelnde Wille derjenigen, die die Entscheidungsmacht darüber haben, Frauen emporkommen zu lassen. Und Motivation bestimmt bekanntlich das Handeln.

Deshalb sollte man die Entscheiderebene selbst ins rechtliche Visier nehmen. Dafür gibt es zwei Ansatzpunkte:

\section{ANREIZSYSTEME SCHAFFEN}

Wenn es mangelnde Motivation ist, Frauen den Aufstieg in höhere berufliche Positionen zu ermöglichen, dann kann man versuchen, die Motivation durch Anreize zu stimulieren und in die gewünschte Richtung zu lenken. So könnten Bonuspunkte, die Managergehälter nicht nur wegen guter Steigerung der Renditen, sondern wegen guter Erfolge bei der Besetzung auch leitender Positionen mit Frauen aufpolstern, vielleicht dazu bewegen, mit etwas mehr Engagement bei Stellenbesetzungen nach geeigneten Frauen Ausschau zu halten. Zwar kann die Zahlung solcher Zuschläge schwerlich erzwungen werden. Eine rechtliche Anregung dazu, verbunden mit einer günstigen steuerlichen Berücksichtigung dieser Ausgaben, könnte jedoch nachhelfen, sich mit solchen Belohnungen von Erfolgen bei der Frauenförderung anzufreunden. Auch das Vergaberecht könnte im Sinne der Frauenförderung genutzt werden: Unternehmen, die eine erfolgreiche Frauenförderung nicht nachweisen können, fallen im Rennen um lukrative Aufträge zurück.

\section{QUOTENREGELUNGEN INSTALLIEREN}

Der zweite, unmittelbarer wirkende und damit noch eher Erfolg versprechende Weg ist, ein Stühlerücken auf der Entscheiderebene anzuordnen und Frauenquoten für die Besetzung von Unternehmensgremien vorzugeben. Nach langer Beschäftigung mit diesem Thema ist mir bewusst: Quoten sind ein rotes Tuch, das männliche Seelen in Wallung bringt und vielfach auch Frauen entrüstet. Sie wollen nicht wegen der Quote, sondern wegen ihrer Fähigkeiten auserkoren werden. Dabei übersehen sie jedoch, dass Männer wie selbstverständlich ihre, in Spitzenpositionen fast an $100 \%$ heranreichende Quote ohne Infragestellung ihrer Befähigung in vollem Umfang in Anspruch nehmen. Deshalb nur keine Scheu, die Quote ist ein opportunes, ja notwendiges Mittel, Frauen die ihnen auch in den vorderen Reihen der Arbeitswelt gebührenden Plätze einzuräumen. In Norwegen wird dieses rechtliche Instrumentarium bei Aufsichtsräten von Unternehmen schon erfolgreich zum Einsatz gebracht. Aber auch und besser noch kann es bei Vorstandspositionen angewendet werden. Diese „Spitzenquote“ hat den Vorteil der Effizienz. Denn sie wirkt sich nicht nur auf die Zusammensetzung des Entscheidungsgremiums selbst aus, sondern kann infolge die Personalpolitik der Unternehmen insgesamt in Richtung einer stärkeren Berücksichtigung von Frauen beeinflussen. Mehr Frauen in Entscheidungsgremien sind zwar noch keine Garantie für eine bessere Unternehmenspolitik. Es hat sich aber erwiesen, dass Frauen in Füh- rungspositionen durchaus einen höheren Sensus für noch bestehende und zu behebende Benachteiligungssituationen in der Arbeitswelt besitzen.

\section{GESELLSCHAFTLICHEN DRUCK AUSÜBEN}

Die Gleichstellung von Frauen und Männern im Arbeitsleben ist also durchaus möglich. Doch es bleibt am Ende die Frage, wer sich dafür stark macht und wie man im Parlament eine Mehrheit findet, die bereit ist, die entsprechenden Instrumentarien per Gesetz zum Einsatz zu bringen. Die Lobby der Wirtschaft, die sich dagegen zur Wehr setzt, hat es bislang geschafft, die jeweiligen Regierungen und die sie stützende Parlamentsmehrheit für sich einzunehmen und sie davon zu überzeugen, die Finger davon zu lassen. Das gilt leider auch für die rot-grüne Regierung unter Gerhard Schröder, die vor der Wirtschaft in Sachen Gleichstellungsgesetz eingeknickt ist. Da hilft es wenig, wenn die SPD nun in der Opposition verkündet, sich des Themas wieder annehmen zu wollen. Dennoch sind alle Bündnispartner willkommen, die sich über Lippenbekenntnisse hinaus für solche Gesetzesvorhaben einsetzen. Das gilt auch für die Gewerkschaften. Letztlich aber können die Frauen auch hier nur sich selber helfen, wie die Geschichte lehrt. Nur wenn sie sich wieder einmal zusammenschließen, wenn sie die Forderung nach Gleichstellung im Arbeitsleben, nach Lohngleichheit und nach der Besetzung auch von Spitzenpositionen in der Wirtschaft mit Frauen auf ihre Fahnen schreiben und wenn sie massiv und öffentlich Druck ausüben, wie dies bei der Durchsetzung des Frauenwahlrechts und der Verankerung des Gleichberechtigungsgebots in unserer Verfassung der Fall war, dann wird sich hier Entscheidendes bewegen. Frauen verfügen über die Hälfte der Wählerstimmen, vereint haben sie Power. Dessen müssen sie sich nur wieder bewusst werden, im Ziel sich einig sein und sich dann bewegen, frauenbewegen. So kann es erneut gelingen. 